\title{
Migraine in menopausal women: a systematic review
}

\author{
This article was published in the following Dove Press journal: \\ International Journal of Women's Health \\ 20 August 2015 \\ Number of times this article has been viewed
}

\section{Patrizia Ripal \\ Raffaele Ornello' \\ Diana Degan' \\ Cindy Tiseo' \\ Janet Stewart ${ }^{2}$ \\ Francesca Pistoia' \\ Antonio Carolei' \\ Simona Saccol \\ 'Department of Applied Clinical Sciences and Biotechnology, Institute of Neurology, University of L'Aquila, L'Aquila, Italy; ${ }^{2}$ Psychology Division, School of Natural Sciences, University of Stirling, Scotland, UK}

Correspondence: Simona Sacco Department of Applied Clinical Sciences and Biotechnology, Institute of Neurology, University of L'Aquila, via Vetoio, 67100 L'Aquila, Italy

Tel +390862 433552

Email simona.sacco@cc.univaq.it

\begin{abstract}
Evidence suggests that migraine activity is influenced by hormonal factors, and particularly by estrogen levels, but relatively few studies have investigated the prevalence and characteristics of migraine according to the menopausal status. Overall, population-based studies have shown an improvement of migraine after menopause, with a possible increase in perimenopause. On the contrary, the studies performed on patients referring to headache centers have shown no improvement or even worsening of migraine. Menopause etiology may play a role in migraine evolution during the menopausal period, with migraine improvement more likely occurring after spontaneous rather than after surgical menopause. Postmenopausal hormone replacement therapy has been found to be associated with migraine worsening in observational population-based studies. The effects of several therapeutic regimens on migraine has also been investigated, leading to nonconclusive results. To date, no specific preventive measures are recommended for menopausal women with migraine. There is a need for further research in order to clarify the relationship between migraine and hormonal changes in women, and to quantify the real burden of migraine after the menopause. Hormonal manipulation for the treatment of refractory postmenopausal migraine is still a matter of debate.
\end{abstract}

Keywords: headache, female, menstrual cycle, sex hormones

\section{Introduction}

Migraine is a primary headache disorder with two major subtypes, ie, migraine without aura (MO) and migraine with aura (MA). MO is a clinical syndrome characterized by headache with specific features and associated symptoms (unilateral location, pulsating quality, moderate or severe intensity, aggravation by routine physical activity, and association with nausea and/or photophobia and phonophobia); while MA is primarily characterized by transient focal neurological symptoms that usually precede or sometimes accompany a headache with migrainous features. ${ }^{1}$

Migraine occurs in both sexes, but it is predominantly found in females, with a cumulative lifetime prevalence of $43 \%$ in women and $18 \%$ in men. ${ }^{2}$ There is strong evidence linking migraine with the hormonal status of women, and both the timing and frequency of migraine attacks are influenced by hormone-related events. Migraine occurrence may be influenced by the varying hormonal status of women through menarche, menstruation, pregnancy, and menopause, as well as use of oral contraceptives and hormone replacement therapy (HRT).

Migraine usually starts after menarche, occurs more frequently during menstruation, and ameliorates during pregnancy and after menopause. ${ }^{3}$ The "estrogen withdrawal" hypothesis, more than 40 years after having been proposed for the first time, is still the most commonly referred to, in order to explain the mechanism through which migraine is triggered by ovarian hormones. ${ }^{4}$ According to this hypothesis, 
migraine is triggered by the sudden decline in estrogen levels occurring immediately before menses and during the transition toward menopause or in the early postmenopausal period. ${ }^{3,5}$ Menopause is a normal physiological event, defined as the final menstrual period and reflecting loss of ovarian follicular function. ${ }^{6}$ The menopausal transition, known as perimenopause, is associated with fluctuating ovarian function. Through a process of continuous depletion called atresia, the human ovary steadily loses follicles; as fewer and fewer ovarian follicles remain, the anterior pituitary gland attempts to compensate by releasing more follicle-stimulating hormone (FSH), which stimulates a recruited follicle to grow and produce estrogen. Chronic elevation of FSH makes the follicular cells insensitive to FSH leading to failure of the final maturation of ovarian follicles (anovulatory cycles). Transient increases in estradiol can occur during this time as surges of FSH stimulate the remaining follicles, but the luteal progesterone decreases as result from declining dominant follicle quality. Once no more follicles remain for stimulation, estradiol levels also decrease and menstruation ceases completely. ${ }^{7}$ Thus, while the perimenopausal period is characterized by considerable fluctuations of estrogen and progesterone levels, greater than those occurring during the normal phases of menstrual cycle in the fertile period, menopause is characterized by hormonal stability due to decline of estrogen and progesterone production by the ovaries. Spontaneous or natural menopause is recognized retrospectively after 12 months of amenorrhea, ${ }^{8}$ occurring on average at the age of 51 years, with an age range of 40-60 years. ${ }^{9}$ Induced menopause refers to the cessation of menstruation that occurs after either bilateral oophorectomy or iatrogenic ablation of ovarian function (such as by chemotherapy or pelvic radiation). ${ }^{10}$

Therapeutic use of exogenous hormones may influence migraine course; regular use of those hormones may be associated with the development of "exogenous hormoneinduced headache". ${ }^{1}$ On the other hand, according to the theory of "estrogen withdrawal headache", headache or migraine may be caused by cessation of a course of exogenous estrogens. Many different cellular actions of estrogens have been described, including effects on vascular tone; the hormonal changes are often responsible for the clinical vasomotor signs and symptoms, such as hot flushes, and may favor migraine. While estrogens induce vasodilation through mechanisms involving the arterial endothelium and through endothelial-independent actions, progesterone has varying effects on arterial tone, including induction of vascular smooth muscle relaxation as well as induction of smooth muscle constriction. ${ }^{11} \mathrm{~A}$ link between hormonal imbalance and migraine may stem from the opposing roles of estrogen and progesterone within the brain; indeed, while estrogen stimulates neural excitability, progesterone exhibits inhibitory actions in central neurons. Therefore, imbalance between these neuromodulatory hormones may give rise to physiological conditions that alter susceptibility to migraine. ${ }^{12}$ Although the focus has been on estrogen, we cannot exclude that other factors may contribute to migraine course and menopause. Estrogen is associated with increased production of serotonin, reduced serotonin reuptake, and decreased serotonin degradation. The consequent cyclical changes in serotonin levels in trigeminal ganglia could contribute to the selective response to estrogen withdrawal. Given that serotonin has also been implicated in the pathophysiology of vasomotor symptoms, it is interesting to speculate on a common pathophysiology. ${ }^{13}$

This paper reviews the available evidence documenting the relationship of menopause and migraine.

\section{Search strategy and selection criteria}

Data for this review were obtained through searches in BIOSIS, the Cochrane database, Embase, Google scholar, ISI Web of Science, and Medline, with a timeframe from 1960 up to December 2014. The search terms included "migraine" OR "headache" AND ("women", "menopause", "perimenopause", "menstruation", "hormonal fluctuations", "sex hormones", "estrogens", "hormone replacement therapy"). We also searched reference lists of identified articles and papers quoting identified articles. No language restrictions were applied. Cohort studies, case-control studies, and clinical trials evaluating the association between menopause and migraine in either hospital-based or population-based settings were included in the review. Studies dealing with the pathophysiological links between migraine and hormones, and letters, reviews and, comments related to this topic were also considered for the discussion but not included in the systematic review.

\section{Results}

Out of 6,326 records, we considered 46 papers after title and abstract review. Six more papers were retrieved from the reference lists of identified articles. We finally included 22 studies $^{14-35}$ in our review.

In this review, we discuss each issue addressed by the studies in a single paragraph. The characteristics of the included studies are summarized in Tables 1 and 2. 


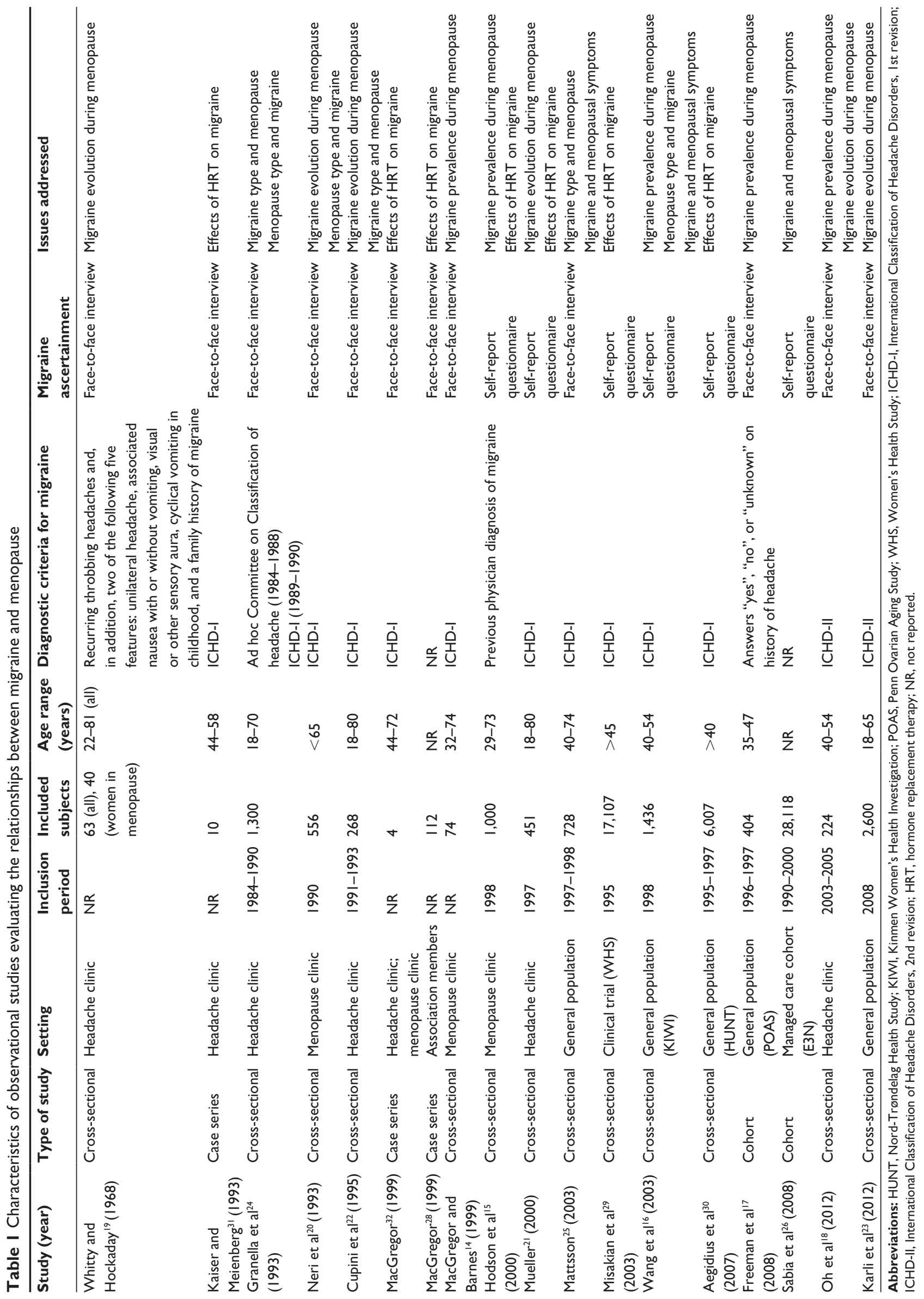




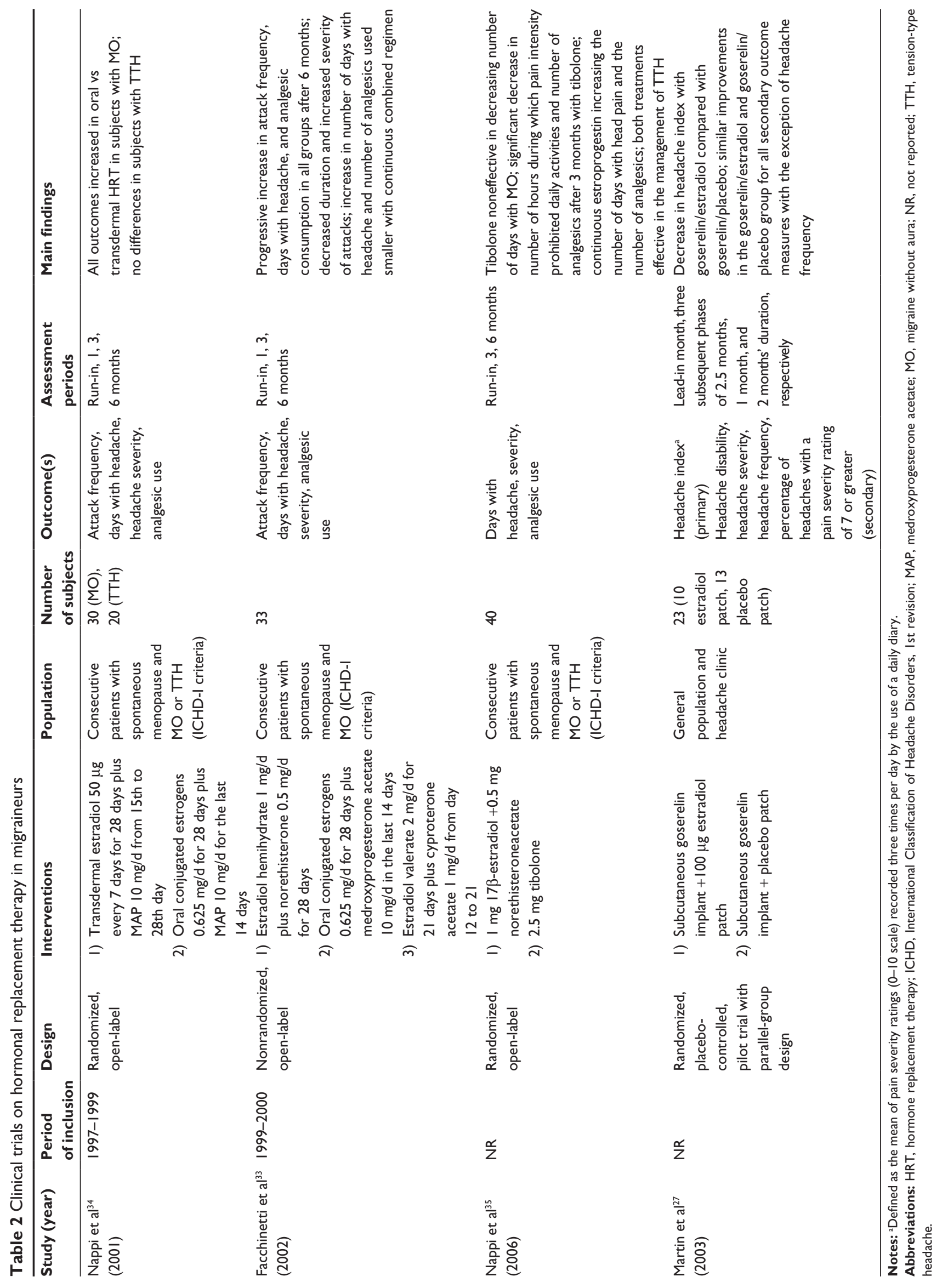




\section{Prevalence of migraine during menopause}

Reported migraine prevalence during menopause ranges from $10 \%$ to $29 \% .^{14-18}$ Prevalence data are not dissimilar among studies, despite heterogeneity in design, settings, number and age of subjects included, and diagnostic criteria for migraine. An early questionnaire survey performed in women attending a menopause clinic found that $57 \%$ of women reported headache and $29 \%$ reported migraine in the 3 months preceding the visit. ${ }^{14}$ In that study, migraine was also associated with significant disability: $80 \%$ of women reported that attacks occurred more than once a month, 75\% reported severe attacks, and 50\% reported that attacks lasted longer than 1 day. A further questionnaire survey performed on 1,000 women, aged 29-73 years, attending a menopause clinic, showed that $85 \%$ of women presented with recurrent headache; among them, $73 \%$ experienced headache more than once per month and $24 \%$ had migraine. ${ }^{15}$ Those two studies did not report details according to the menopausal stages, ${ }^{14,15}$ but other studies reported this information. ${ }^{16-18}$ In a cross-sectional study of 1,436 menopausal women aged 40-54 years, $69 \%$ reported having at least one headache in the previous year, and $16.5 \%$ were diagnosed with migraine according to International Headache Society criteria. Women with spontaneous menopause reported a migraine prevalence of $11 \%$, while premenopausal and perimenopausal women reported a prevalence of $17 \%$ (odds ratio [OR], 0.6; 95\% confidence interval $[\mathrm{CI}], 0.4-0.9 ; P=0.03) .{ }^{16}$ In a populationbased cohort study with 404 premenopausal women aged 35-47 years assessed prospectively for 9 years, 34\% of premenopausal women but only $24 \%$ of postmenopausal women reported headache at the end of follow-up. Headache prevalence was lower in postmenopause (OR, 0.60; 95\% CI, 0.42-0.83; $P=0.003$ ), but not in perimenopause, compared with reproductive period. ${ }^{17}$ A cross-sectional study investigating the headache characteristics of 229 middleaged women (aged 40-54 years) consecutively attending a headache clinic showed a comparable prevalence of migraine among women in the pre-, peri-, and postmenopausal period. The proportion of patients with a brief migraine history ( $<6$ months) was significantly higher in the perimenopausal group (41\%) than in the premenopausal $(13 \%)$ or postmenopausal (17\%) groups. ${ }^{18}$ In summary, data from the available studies suggest that migraine prevalence remains stable or increases in perimenopause and declines after menopause compared with reproductive period. Population-based studies supported this trend more than those performed in hospital settings.

\section{Migraine evolution during menopause}

To date, there are very few large, prospective, populationbased cohort studies to determine the true effect of menopause on migraine. The available studies are mostly retrospective and questionnaire-based. A retrospective study evaluating the course of $\mathrm{MO}$, by means of a questionnaire, in 47 postmenopausal women aged 43-67 years, reported an improvement in 24 women (62\%), a worsening in $7(18 \%)$, and no changes in $8(20 \%)$, while $8(17 \%)$ had new onset of headache with menopause. ${ }^{20} \mathrm{~A}$ cross-sectional study carried out on 268 women ( 36 postmenopausal) with migraine referring to a single headache center found an improvement of migraine with spontaneous menopause. ${ }^{22}$ Conversely, another study involving 53 female migraineurs retrospectively assessed 15-20 years after their first attendance at a neurology ward did not find any consistent change in attacks after menopause in $64 \%$ of the participants. Moreover, a worsening of migraine status was reported in slightly more cases $(21 \%)$ than an improvement or complete remission $(14 \%) .{ }^{19} \mathrm{~A}$ further retrospective study performed in 451 women with MA or MO aged 18-80 years did not find migraine improvement during menopause. In fact, among 160 women in the menopause, the course of migraine was improving in 41 patients (24\%), worsening in 60 (36\%), and unchanged in $67(40 \%) .{ }^{21}$ More recently, an analysis of headaches in perimenopausal patients attending a headache clinic and not receiving exogenous hormone treatment showed that current headaches were reported as new-onset by $48 \%$ of subjects, aggravations of prior headaches by $34 \%$ of subjects, and unchanged from prior headaches in $18 \%$ of subjects. ${ }^{18}$ Meanwhile in a cross-sectional, population-based study evaluating 1,333 women aged $18-65$, only $17 \%$ of migraineurs reported improvement during menopause, ${ }^{23}$ although the low number of menopausal subjects may have affected the results. In general, according to populationbased studies, menopause may bring an improvement of migraine course, while according to the studies performed in hospital settings, migraine may remain stable or even worsen following menopause.

\section{Migraine type and menopause}

There is some evidence to suggest that MA and MO are differently influenced by women's reproductive life events, ${ }^{24,25}$ although, to date, available data are scarce and too heterogeneous to confidently accept that such a difference exists. In a cross-sectional study that included 10 postmenopausal women with MA and 26 with MO consecutively referring to a Headache Center, no difference was found between the two 
groups of patients with regard to postmenopausal migraine onset and migraine course during menopause. ${ }^{24}$ A subsequent population-based study performed on 728 women (301 postmenopausal) aged 40-74 years showed a decreased risk of MO in postmenopausal women as compared with women in their fertile period (OR, 0.47; 95\% CI, 0.24-0.86). After adjusting for age differences, however, the association was not statistically significant. In that same study, MA was not associated with the menopausal transition. Notably, time since menopause was a predictor of MO improvement in postmenopausal women. ${ }^{25}$ In summary, the available literature suggests that MO is more influenced by hormones than MA, not only in the fertile period but also during and after the menopause.

\section{Effect of spontaneous and of induced menopause on migraine}

The available data are relatively concordant in showing that spontaneous menopause is associated with a more evident improvement of migraine compared with induced menopause. ${ }^{16,20,24}$ In a postmenopausal outpatient survey, migraine improvement occurred in $67 \%$ of women after spontaneous menopause and in 33\% after surgical menopause; notably, migraine worsened in $9 \%$ of women after spontaneous menopause and in $67 \%$ after surgical menopause. ${ }^{20}$ A retrospective study of 164 postmenopausal women with MO showed that worsening of migraine was significantly more frequent after surgical menopause than after spontaneous menopause; migraine improvement occurred only in women with spontaneous menopause. ${ }^{24}$ Regrettably, that study did not clearly define the aspects of migraine worsening (increased frequency, severity, or duration of attacks). In a subsequent study, a higher migraine prevalence was found in women with surgical menopause than in those with spontaneous menopause, among patients with a history of premenstrual syndrome (44\% vs $7 \% ; P<0.0001$ ), while surgical menopause did not significantly affect migraine prevalence among patients without a history of premenstrual syndrome (16\% vs $12 \% ; P=0.35) .{ }^{16}$ In summary, data from the available studies suggest that migraine prevalence remains stable or increases in perimenopause and declines after menopause compared with the reproductive period. Population-based studies supported this trend more than those performed in hospital settings.

\section{Migraine and menopausal symptoms}

The association between migraine and menopausal symptoms, such as mood changes, poor sleep, irritability, and concentration difficulties, is unclear since data from available studies are conflicting. ${ }^{16,25,26}$ A population-based retrospective study of 728 Swedish women reported no association between the presence of migraine and menopausal symptoms. ${ }^{25}$ Conversely, the results of a subsequent cohort study of 28,118 French women suggested that the risk of onset of any menopausal symptom was higher in subjects with a history of migraine (hazard ratio [HR], 1.11; 95\% CI, $1.07-1.16 ; P=0.007) .{ }^{26}$ Another study analyzed the association between menopausal vasomotor symptoms (hot flushes, night sweating) and migraine and found that subjects with either hot flushes (22\% vs 16\%; OR, 1.5; 95\% CI, 1.0-2.2; $P=0.044)$ or night sweating ( $28 \%$ vs $16 \%$; OR, $2.1 ; 95 \% \mathrm{CI}$, $1.3-3.4 ; P=0.003$ ) had a higher migraine prevalence than those without these symptoms. ${ }^{16}$ The differences among studies may be explained either by the confounders assessed in their adjusted models or by a different effect of migraine on each menopausal symptom. Different study designs and sample sizes may also have contributed to between-studies differences in the reported results.

\section{Effects of menopausal HRT on migraine}

Women with menopause occurring before 45 years of age are at increased risk for long-term health consequences, including premature cardiovascular disease, mortality, Parkinsonism, cognitive decline, depression, anxiety, and sexual dysfunction. ${ }^{36}$ HRT is typically recommended for these women until the age of expected natural menopause in order to reduce the risks as well as to relieve menopausal symptoms and to slow down the decrease in bone density that occurs after menopause.

HRT has a variable effect on migraine. A survey questionnaire performed on 112 perimenopausal or postmenopausal female members of the British Migraine Association using HRT (oral or transdermal conjugated estrogens combined with cyclical oral progestogen) showed a trend toward greater improvement of migraine in women using transdermal estrogen compared with oral conjugated estrogens and found that headache was often associated with the progestogen phase of cyclical therapy. ${ }^{28}$ Two studies reported an increased risk of migraine among users of HRT. ${ }^{29,30}$ Among 17,107 postmenopausal women selected from the Women's Health Study, 62\% were current users of HRT and 39\% had never used HRT, and $11 \%$ of all postmenopausal women - both users and nonusers - experienced migraine. Current HRT use was associated with higher risk of migraine than nonuse (OR, 1.42; 95\% CI, 1.24-1.62), both for users of estrogen alone (OR, 1.39; 95\% CI, 1.14-1.69) and users of estrogen plus progestin (OR, 1.41; $95 \%$ CI, 1.22-1.63). There were no significant differences in 
the risk of migraine headache in users of cyclic versus continuous doses of estrogens, and no dose-response relationship was reported. ${ }^{29}$ Among 5,507 postmenopausal women selected from the Nord-Trøndelag Health Study (HUNT) study, 2,375 (43\%) reported use of HRT ( $26 \%$ previous, $20 \%$ local, $54 \%$ systemic). Migraine prevalence in the groups of never, previous, and present (local or systemic) use of HRT was $49 \%, 11 \%$, and $40 \%$, respectively. An association was found between migraine and current use of HRT (OR, 1.6; 95\% CI, 1.4-1.9), showing that migraine was more common in HRT users as compared with nonusers. ${ }^{30}$

Other studies showed that worsening of migraine in menopause may be a predictor of migraine worsening on HRT. ${ }^{15,21}$ In a questionnaire survey performed in a UK menopause clinic, women were asked about headache and were invited to note its severity on a visual analog scale. Women with improved or unchanged headache during menopause generally followed a similar pattern on HRT use, however, the women with headache worsening during menopause showed a variable response to HRT. The probability of headache worsening at menopause and with use of HRT was significantly increased with reported history of migraine. ${ }^{15}$ Moreover, a retrospective study conducted on 451 female migraineurs found an association between self-reported worsening of headache on HRT and worsening of headache during menopause $(67 \%$ vs $27 \%, P<0.01){ }^{21}$

HRT may have adverse effects on MA. Among ten menopausal women using estrogen cutaneous patches and consecutively referring to a Headache clinic, three had MA worsening, three had new-onset visual aura without migraine, one had new-onset MA, and three switched from MO to MA. ${ }^{31}$ Another study, in which consecutive patients referring to a menopause clinic were assessed, suggested that HRT could lead to migraine worsening. ${ }^{32}$ Higher doses of estrogen replacement therapy may be more likely to lead to the development of aura symptoms; lowering the dosage or switching to another type of estrogen replacement may lead to an abatement of aura symptoms. ${ }^{32}$ It should be noted, however, that as those studies evaluated consecutive patients in tertiary clinics, the possibility of selection bias cannot be excluded. As regards MO, one study showed that postmenopausal women on HRT did not have a different prevalence of MO compared with premenopausal women, while postmenopausal women not on HRT had a lower prevalence of MO compared with premenopausal women. ${ }^{25}$

Some clinical trials have investigated the relationship between various HRT regimens and migraine (Table 2). ${ }^{27,33-35}$
The first randomized trial performed in women with $\mathrm{MO}$ found that both frequency of attacks and days with headache significantly increased during HRT in the subgroup consuming oral formulation, while no changes in the same parameters were found in the group taking transdermal treatment. The severity of migraine was unaffected by HRT, while analgesic consumption was significantly increased in the subgroup on oral treatment. ${ }^{34} \mathrm{~A}$ subsequent trial found a significant increase in attack frequency, days with headache, and analgesic consumption in postmenopausal women receiving HRT; duration of attacks decreased, whereas severity worsened. The increase in number of days with headache and number of analgesics used was smaller in the group receiving a continuous combined regimen than in cyclical regimens. ${ }^{33}$ Another randomized trial found that tibolone, a synthetic steroid hormone with estrogenic, progestogenic, and androgenic properties did not affect the number of days with MO, but significantly reduced the number of hours during which pain intensity prohibited daily activities and the number of analgesics consumed as compared with oral continuous HRT. ${ }^{35}$

Induction of menopause has been proposed as a therapy in patients with refractory migraine, with the rationale of minimization of hormonal fluctuations. Refractory migraine has been defined as a migraine disorder that causes significant interference with function or quality of life despite modification of triggers, lifestyle factors, and adequate trials of acute and preventive (at least two) medicines with established efficacy. ${ }^{37}$ In a placebo-controlled trial, 23 such patients were randomized to receive chemical oophorectomy either with goserelin - a GnRH agonist - and estrogen add-back therapy, or with goserelin and placebo, with a follow-up period of 6.5 months. The women who received goserelin and estrogen add-back therapy had less pain intensity after 3 months than those receiving goserelin and placebo. ${ }^{27}$ Overall, it should be noted that the small sample size, the lack of placebo groups, and the inappropriate or unclear endpoint definitions in these studies limit their ability to determine to what extent HRT is beneficial, or otherwise, for migraineurs.

\section{Discussion}

Our systematic review showed that the available studies on migraine course in menopause are relatively few, heterogeneous, and with conflicting results. Migraine improvement after menopause is a widely accepted notion, mostly because of common clinical experience rather than due to the presence of large epidemiological studies providing robust evidence for this. Although in many patients migraine ameliorates 
with the menopause, during the transition to menopause, migraine attacks can become more severe and frequent due to fluctuating and changing hormonal levels in this period. Unfortunately, no longitudinal studies have been conducted to determine the exact effect of the perimenopausal period on migraine.

It is of note that an increased prevalence of migraine in perimenopausal women and a decreased prevalence in postmenopausal women, compared with premenopausal women, has been found only in community-based studies, ${ }^{16}$ while studies performed in headache clinics do not show significant differences between pre- and postmenopause. ${ }^{18}$ This may be because Headache clinics select patients with more severe headache compared with the general population. The same selection bias may occur for migraine evolution during menopause, with the highest percentages of migraine worsening coming from Headache clinics ${ }^{18,20,24}$ and the highest percentages of migraine improvement reported by population-based studies. ${ }^{17,26}$ The different results may also be due to differences in study populations, where the proportion of women in perimenopause and menopause differ. Dissimilar age ranges in menopausal stages may have also contributed to inconsistencies among the study results.

"Headache attributed to exogenous hormones" and "estrogen withdrawal headache" have already been internationally coded as headache diagnoses. ${ }^{1}$ The fact that both estrogen addition and estrogen withdrawal may trigger a headache with migrainous features suggests that migraine is sensitive to hormonal fluctuations rather than to the amount of circulating hormones. There is some suggestion that hormonally sensitive women (eg, those who have experienced migraine changes in conjunction with previous hormonal events such as menstruation, pregnancy, and use of oral contraceptives) are more likely to have migraine worsening in the perimenopause. Women with a history of menstrual migraine may be more vulnerable to exacerbation of migraine during the hormonally unstable perimenopausal period. ${ }^{16}$ The transition from reproductive to nonreproductive phase is the result of a progressive reduction in female hormonal production by the ovaries and usually lasts $4-8$ years. ${ }^{38}$ Although the perimenopausal period is characterized by considerable fluctuations of estrogen and progesterone levels, greater than those occurring during the normal phases of menstrual cycle in the fertile period, menopause is characterized by hormonal stability due to the decline of estrogen and progesterone production by the ovaries. ${ }^{3}$ During perimenopause, the slower these hormonal changes occur, the greater the improvement of migraine.
The achievement of hormonal stability on menopause is positively associated with migraine improvement. ${ }^{17,25}$

The type of menopause - spontaneous or induced - has a substantial effect on migraine. In spontaneous menopause, the likelihood of migraine improvement has been reported to be as high as $50 \%-60 \%,{ }^{39}$ although with high variability among studies. Surgically induced menopause (ie, oophorectomy with or without hysterectomy) appears to be associated with worsening of migraine in a substantial percentage of women. ${ }^{20}$ However, the numbers vary widely among studies. Migraine worsening occurs even with retention of the ovaries or postsurgical estrogen therapy. ${ }^{40}$ The reason for worsening is unclear, but it may be related to the abrupt, large change in hormones and/or the use of supplementary estrogen following surgery. Chemical oophorectomy with estrogen therapy has been performed for the prevention of refractory migraine ${ }^{36}$ and has been suggested as a way of selecting women for surgical menopause. However, this approach is unproven. In women suffering from severe and disabling migraines which improve after pharmacologically induced menopause, hysterectomy with bilateral oophorectomy may be considered, but its effectiveness has not been demonstrated.

The effect of HRT on migraine is unpredictable. Either the usual contraceptive regimens or estrogen supplements can be used during the perimenopause. In addition to the use of hormonal formulations different from contraceptive regimens, the choice of estrogen supplements for the specific treatment of climacteric symptoms, including migraine, has been advocated. ${ }^{41}$ The goal of hormonal treatment regimens for migraine is minimization of estrogen fluctuations. However, the erratic endogenous estrogen levels of perimenopause may increase the uncertainty of exogenous dosing. ${ }^{42}$ HRT may have variable effects depending on the route of administration, type of estrogen, and type of administration (cyclical vs continuous). Transdermal preparations may be less likely to exacerbate migraine, but this has not been consistently shown. ${ }^{41,43}$ Progesterone supplementation may be effective for treating menstrual migraine by suppressing menstruation. In general, progesterone dosing does not seem to change migraine frequency, duration, or severity. ${ }^{41} \mathrm{~A}$ better understanding of the mechanisms linking migraine and hormonal fluctuations in menopause could lead to better treatment for patients suffering from migraine.

From a clinical perspective, in the absence of conclusive results, no specific therapies can be indicated for menopausal migraine. Most medications used in patients 
in reproductive age, including both acute medications (as nonsteroidal anti-inflammatory drugs and triptans) and preventive medications (as $\beta$-blockers, calcium channel blockers, tricyclic antidepressants, selective serotonin reuptake inhibitors, and antiepileptic drugs), are also useful in healthy women in that age group. In menopausal women, additional factors may influence the onset or worsening of headache disorders, including migraine; among these factors, stress plays a major role. ${ }^{15,17}$ Some migraine preventative drugs act on biological stress mechanisms and may be effective in migraineurs during menopause.

In migraineurs, HRT use may be challenging because of the potential worsening of migraine. Markers of "hormonal sensitivity" have been proposed, including menstrual migraine or onset at menarche, ${ }^{21}$ history of improvement during pregnancy, ${ }^{21}$ premenstrual syndrome, ${ }^{16}$ and surgical menopause. ${ }^{16,20,24}$ Recognition of these markers in migraineurs could help in planning the cautious use of HRT in order to avoid migraine worsening. Migraine worsening during menopause is associated with worsening also on HRT..$^{15,21}$ This association may also direct the therapeutic decisions of both clinicians and their patients. There is a paucity of scientific evidence to guide decision making regarding HRT for women who undergo surgical menopause and experience a worsening of headaches but need to take estrogen replacement to reduce long-term health consequences of premature estrogen deficiency. Further longitudinal studies should be performed to clearly identify markers of "menopausal migraine" and "HRT-associated migraine" and to plan specific treatments. To date, migraine does not represent a contraindication for HRT; in any case, it is reasonable to administer the lowest effective doses. ${ }^{13}$ Moreover, there are also health risks associated with HRT use, such as the risk of cancer and cardiovascular diseases. ${ }^{44}$ Due to evidences suggesting that migraine, particularly MA is associated with an increased risk of ischemic ${ }^{45}$ and hemorrhagic stroke ${ }^{46}$ and myocardial infarction, ${ }^{47}$ we cannot exclude that this risk may be further elevated in the setting of combined estrogen-progesterone contraceptive use. There are no studies that directly assess the risk of stroke in migraineurs using HRT. For these reasons, the control of migraine via hormonal manipulation is still challenging, although the use of HRT to minimize fluctuations in estrogen may be a potentially helpful therapy in some menopausal women, and there is no enough scientific evidence to recommend it.

\section{Disclosure}

The authors report no conflicts of interest in this work.

\section{References}

1. Headache Classification Committee of the International Headache Society (IHS). The International Classification of Headache Disorders, 3rd edition (beta version). Cephalalgia. 2013;33:629-808.

2. Stewart WF, Wood C, Reed ML, Roy J, Lipton RB; AMPP Advisory Group. Cumulaative lifetime migraine incidence in women and men. Cephalalgia. 2008;28:1170-1178.

3. Sacco S, Ricci S, Degan D, Carolei A. Migraine in women: the role of hormones and their impact on vascular diseases. J Headache Pain. 2012; 13(3):177-189.

4. Somerville BW. The role of estradiol withdrawal in the etiology of menstrual migraine. Neurology. 1972;22(4):355-365.

5. Wober C, Brannath W, Schmidt K, et al. Prospective analysis of factors related to migraine attacks: the PAMINA study. Cephalalgia. 2007;27: 304-314.

6. World Health Organization Scientific Group. Research on The Menopause. WHO Technical Report Series, No 670. Geneva, Switzerland: World Health Organization Scientific Group; 1981.

7. Sulak PJ. The perimenopause: a critical time in a woman's life. Int J Fertil Menopausal Stud. 1996;41(2):85-89.

8. World Health Organization Scientific Group. Report of WHO Scientific Group: Research on the Menopause in the 1990's. Geneva, Switzerland: World Health Organization; 1996.

9. Shifren JL, Gass ML. The North American Menopause Society recommendations for clinical care of midlife women. Menopause. 2014;21(10): 1038-1062.

10. Sarrel PM. The differential effects of oestrogens and progestins on vascular tone. Hum Reprod Update. 1999;5(3):205-209.

11. Finocchi C, Ferrari M. Female reproductive steroids and neuronal excitability. Neurol Sci. 2011;32:S31-S35.

12. TeVelde E, Pearson P. The variability of female reproductive aging. Hum Reprod Update. 2002;8:141-154.

13. MacGregor EA. Migraine headache in perimenopausal and menopausal women. Curr Pain Headache Rep. 2009;13:399-403.

14. MacGregor EA, Barnes D. Migraine in a specialist menopause clinic. Climacteric. 1999;2(3):218-223.

15. Hodson J, Thompson J, Azzawi F. Headache at menopause and in hormone replacement therapy users. Climacteric. 2000;3:119-124.

16. Wang SJ, Fuh JL, Lu SR, Juang KD, Wang PH. Migraine prevalence during menopausal transition. Headache. 2003;43:470-478.

17. Freeman EW, Sammel MD, Lin H, Gracia CR, Kapoor S. Symptoms in the menopausal transition: hormone and behavioral correlates. Obstet Gynecol. 2008;111:127-136.

18. Oh K, Jung KY, Choi JY, Seo WK, Park KW. Headaches in middle-aged women during menopausal transition: a headache clinic-based study. Eur Neurol. 2012;68(2):79-83.

19. Whitty CW, Hockaday JM. Migraine: a follow-up study of 92 patients. Br Med J. 1968;1(5594):735-736.

20. Neri I, Granella F, Nappi R, Manzoni GC, Facchinetti F, Genazzani AR. Characteristics of headache at menopause: a clinico-epidemiologic study. Maturitas. 1993;17:31-37.

21. Mueller L. Predictability of exogenous hormone effect on subgroups of migraineurs. Headache. 2000;40:189-193.

22. Cupini LM, Matteis M, Troisi E, Calabresi P, Bernardi G, Silvestrini M. Sex-hormone-related events in migrainous females. A clinical comparativestudy between migraine with aura and migraine without aura. Cephalalgia. 1995;15:140-144.

23. Karli N, Baykan B, Ertaş M, et al. Impact of sex hormonal changes on tension-type headache and migraine: a cross-sectional population-based survey in 2,600 women. J Headache Pain. 2012;13(7): $557-565$.

24. Granella F, Sances G, Zanferrari C, Costa A, Martignoni E, Manzoni GC. Migraine without aura and reproductive life events: a clinical epidemiological study in 1300 women. Headache. 1993;33: 385-389.

25. Mattsson P. Hormonal factors in migraine: a population-based study of women aged 40 to 74 years. Headache. 2003;43:27-35. 
26. Sabia S, Fournier A, Mesrine S, Boutron-Ruault MC, Clavel-Chapelon F. Risk factors for onset of menopausal symptoms. Results from a large cohort study. Maturitas. 2008;60:108-121.

27. Martin V, Wernke S, Mandell K, et al. Medical oophorectomy with and without estrogen add-back therapy in the prevention of migraine headache. Headache. 2003;43:309-321.

28. MacGregor A. Effects of oral and transdermal estrogen replacement on migraine. Cephalalgia. 1999;19(2):124-125.

29. Misakian AL, Langer RD, Bensenor IM, et al. Postmenopausal hormone therapy and migraine headache.J Womens Health (Larchmt). 2003;12(10): 1027-1036.

30. Aegidius KL, Zwart JA, Hagen K, Schei B, Stovner LJ. Hormone replacement therapy and headache prevalence in postmenopausal women. The Head-HUNT study. Eur J Neurol. 2007;14(1):73-78.

31. Kaiser HJ, Meienberg O. Deterioration or onset of migraine under oestrogen replacement therapy in the menopause. J Neurol. 1993;240(3): 195-196.

32. MacGregor A. Estrogen replacement and migraine aura. Headache. 1999;39(9):674.

33. Facchinetti F, Nappi RE, Tirelli A, Polatti F, Nappi G, Sances G. Hormone supplementation differently affects migraine in postmenopausal women. Headache. 2002;42(9):924-929.

34. Nappi RE, Cagnacci A, Granella F, Piccinini F, Polatti F, Facchinetti F. Course of primary headaches during hormone replacement therapy. Maturitas. 2001;38(2):157-163.

35. Nappi RE, Sances G, Sommacal A, et al. Different effects of tibolone and low-dose EPT in the management of postmenopausal women with primary headaches. Menopause. 2006;13(5):818-825.

36. Shuster LT, Faubion SS, Sood R, Casey PM. Hormonal manipulation strategies in the management of menstrual migraine and other hormonally related headaches. Curr Neurol Neurosci Rep. 2011;11(2): 131-138.
37. Schulman EA, Peterlin BL, Lake AE 3rd, et al. Defining refractory migraine: results of the RHSIS Survey of American Headache Society members. Headache. 2009;49(4):509-518.

38. Harlow SD, Paramsothy P. Menstruation and the menopause transition. Obstet Gynecol Clin North Am. 2011;38(3):595-607.

39. Bousser MG. Estrogens, migraine, and stroke. Stroke. 2004;35(11 Suppl 1): 2652-2656.

40. MacGregor EA. Migraine and the menopause. J Br Menopause Soc. 2006;12:104-108.

41. Loder E, Rizzoli P, Golub J. Hormonal management of migraine associated with menses and the menopause: a clinical review. Headache. 2007;47:329-340.

42. Lay CL, Broner SW. Migraine in women. Neurol Clin. 2009;27: 503-511.

43. MacGregor EA. Prevention and treatment of menstrual migraine. Drugs. 2010;70:1799-1818.

44. Taylor HS, Manson JE. Update in hormone therapy use in menopause. $J$ Clin Endocrinol Metab. 2011;96(2):255-264.

45. Spector JT, Kahn SR, Jones MR, Jayakumar M, Dalal D, Nazarian S. Migraine headache and ischemic stroke risk: an updated meta-analysis. Am J Med. 2010;123:612-624.

46. Sacco S, Ornello R, Ripa P, et al. Migraine and risk of ischaemic heart disease: a systematic review and meta-analysis of observational studies. Eur J Neurol. 2015;22(6):1001-1011.

47. Sacco S, Ornello R, Ripa P, Pistoia F, Carolei A. Migraine and hemorrhagic stroke: a meta-analysis. Stroke. 2013;44(11):3032-3038.
International Journal of Women's Health

\section{Publish your work in this journal}

The International Journal of Women's Health is an international, peerreviewed open-access journal publishing original research, reports, editorials, reviews and commentaries on all aspects of women's healthcare including gynecology, obstetrics, and breast cancer. The manuscript management system is completely online and includes

\section{Dovepress}

a very quick and fair peer-review system, which is all easy to use. Visit http://www.dovepress.com/testimonials.php to read real quotes from published authors. 